\title{
A Distinctly Interdecadal Signal of Pacific Ocean-Atmosphere Interaction
}

\author{
Oliver W. Frauenfeld \\ Cooperative Institute for Research in Environmental Sciences, University of Colorado, Boulder, Colorado \\ Robert E. Davis and Michael E. Mann \\ Department of Environmental Sciences, University of Virginia, Charlottesville, Virginia
}

(Manuscript received 5 June 2003, in final form 14 October 2004)

\begin{abstract}
A new and distinctly interdecadal signal in the climate of the Pacific Ocean has been uncovered by examining the coupled behavior of sea surface temperatures (SSTs) and Northern Hemisphere atmospheric circulation. This interdecadal Pacific signal (IPS) of ocean-atmosphere interaction exhibits a highly statistically significant interdecadal component yet contains little to no interannual (El Niño scale) variability common to other Pacific climate anomaly patterns. The IPS thus represents the only empirically derived, distinctly interdecadal signal of Pacific Ocean SST variability that likely also represents the true interdecadal behavior of the Pacific Ocean-atmosphere system. The residual variability of the Pacific's leading SST pattern, after removal of the IPS, is highly correlated with El Niño anomalies. This indicates that by simply including an atmospheric component, the leading mode of Pacific SST variability has been decomposed into its interdecadal and interannual patterns. Although the interdecadal signal is unrelated to interannual El Niño variability, the interdecadal ocean-atmosphere variability still seems closely linked to tropical Pacific SSTs. Because prior abrupt changes in Pacific SSTs have been related to anomalies in a variety of physical and biotic parameters throughout the Northern Hemisphere, and because of the persistence of these changes over several decades, isolation of this interdecadal signal in the Pacific Ocean-atmosphere system has potentially important and widespread implications to climate forecasting and climate impact assessment.
\end{abstract}

\section{Introduction}

The cause and nature of interdecadal climate variability is still in large part uncertain. Although decadal fluctuations have been observed in the tropical and extratropical Pacific and the tropical and North Atlantic Ocean, it is unclear whether this low-frequency variability is merely red noise, that is, the slow-response component of the ocean system arising from the random (white noise) forcing of the atmosphere (Hasselmann 1976; Seager et al. 2001) or whether it may represent oceanic or ocean-atmosphere variability with preferred time scales (e.g., Latif and Barnett 1994; Mann and Park 1996; Minobe 1997, 1999). Decadal Pacific Ocean variability has been shown to occur at bidecadal (15-20 yr) and interdecadal (50-70 yr) scales

Corresponding author address: Dr. Oliver W. Frauenfeld, CIRES/NSIDC, University of Colorado, 449 UCB, Boulder, CO 80309-0449.

E-mail: oliverf@kryos.colorado.edu (e.g., Mann and Park 1996; Minobe 1999). Around 1976/77, retrospective analyses indicate that spatial patterns of Pacific sea surface temperature (SST) anomalies exhibited a significant reversal over a short period of time. This "Pacific Climate Shift" (Miller et al. 1994) not only had ramifications for the large marine ecosystems in the North Pacific Ocean (Mantua and Hare 2002), but also coincided with abrupt changes in many climate records, such as tropical Pacific SSTs related to El Niño-Southern Oscillation (ENSO; Guilderson and Schrag 1998), North Pacific SSTs (Miller et al. 1994), the oceanic heat content (Levitus et al. 2000), the radiosonde temperature record of Angell (1999), atmospheric sea level pressure, surface air temperature (Minobe 1997, 1999), etc.

Long-term records of an index of spatial SST patterns called the Pacific (inter) decadal oscillation (PDO) capture this abrupt state shift and also demonstrate a strong tendency for multiyear and multidecadal persistence (Mantua et al. 1997; Mantua and Hare 2002). Given the persistence of these temperature 
anomaly fields and the significant influence of the Pacific Basin on global climate, changes in oceanic conditions like those characterized by the PDO could have a major impact on climate forecasting.

The PDO is defined as the leading principal component (PC) of Pacific Ocean SST departures from monthly mean global average SSTs north of $20^{\circ} \mathrm{N}$ (Zhang et al. 1997). In its positive phase, the PDO SST anomaly pattern is similar to that of the well-known ENSO phenomenon, with abnormally warm water in the tropical eastern Pacific and off the west coast of North America and cooler conditions over the North Pacific. The PDO is not only spatially similar to ENSO, its time series also exhibits significant variability at the interannual ENSO scale and the interdecadal scale (Mantua et al. 1997; Mantua and Hare 2002).

Given the significant interaction between the Pacific Ocean and the atmosphere, it is beneficial to examine statistically the climate patterns that arise from this coupling. Frauenfeld and Davis (2002) employed a combination of principal components analysis (PCA) and canonical correlation analysis (CCA) to isolate unique patterns of Pacific Ocean-atmosphere interaction. Most prominently, the leading SST pattern identified by Frauenfeld and Davis (2002) is very similar to the PDO but exhibits much more pronounced interdecadal persistence. By incorporating hemispheric-scale atmospheric circulation information together with SST variability, apparently different processes are at play at the interdecadal scale than could be gleaned from extratropical SST data alone.

Here, we establish the degree to which the time series of the leading pattern of Pacific Ocean-Northern Hemisphere atmosphere interaction is interdecadal. Given the substantial influence of the Pacific Ocean on hemispheric and global climate, identification of a truly interdecadal Pacific climate signal, coupled with our current level of understanding of interannual Pacific climate variability, could lead to improved prediction of future climate and climate change.

\section{Data and methods}

Monthly $5^{\circ} \times 5^{\circ}$ gridded Kaplan SST anomalies (Kaplan et al. 1998) from January 1949-December 2000 are analyzed for $25^{\circ} \mathrm{S}-65^{\circ} \mathrm{N}$, a domain that includes both the tropical and extratropical Pacific Ocean. The data were standardized at the monthly level relative to their long-term monthly mean and standard deviation to remove any seasonal variability and were weighted by the cosine of latitude to account for varying grid-box size. PCA is applied on the correlation matrix for data reduction and to obtain orthogonal variables for use in subsequent analyses.
To represent the three-dimensional structure of Northern Hemisphere atmospheric circulation, we calculated the position of the monthly circumpolar vortex from January 1949 to December 2000 at every $5^{\circ}$ longitude for the 300-, 500-, and 700-hPa constant pressure levels. The vortex, which represents the location of the central core of the hemispheric midlatitude wind field, has been used effectively for decades to provide a parsimonious measure of atmospheric midlatitude longwave wind flow (e.g., Willett 1949; LaSeur 1954; Angell and Korshover 1977; Markham 1985; Angell 1998; Frauenfeld and Davis 2000, 2002, 2003). The representative contour selection follows the established definition of the vortex and was achieved by selecting the mean representative geopotential height contour for 700,500 , and $300 \mathrm{hPa}$ for each month of the year that represents the area of greatest height change within the core of the westerly flow, that is, the primary baroclinic zone of the 700-, 500-, and 300-hPa vortex, respectively (Burnett 1993). This contour is considered to be the "center contour." To capture more completely the geometry of the Northern Hemisphere circulation field's variability away from the core of the westerly flow, a "southern contour" and a "northern contour" were also selected at each pressure level. These additional contours were chosen to be two-three standard contours south and north of the center contour, thereby characterizing the circulation at lower and higher latitudes. [For complete details of the circumpolar vortex climatology, please refer to Frauenfeld and Davis (2003).]

Atmospheric conditions over most of the Northern Hemisphere are linked with the Pacific Ocean. Both modeling (Barnett et al. 1989) and empirical (Cohen and Entekhabi 1999) studies demonstrate that upstream circulation variability over Eurasia influences Pacific SSTs. Local air-sea feedbacks over the Pacific have been documented (e.g., Alexander et al. 2002), and the interactions between Pacific SSTs and atmospheric circulation in the Pacific-North American region via thermal and moisture fluxes from the sea surface are well established (e.g., Namias 1976). To examine the relationship of Pacific Ocean SST variability and atmospheric circulation, the vortex over the entire Northern Hemisphere is therefore used to characterize circulation variability. As with the Pacific SST field, the vortex data are standardized to remove the seasonal cycle and PCA is again applied for data reduction and orthogonalization.

CCA was then employed on the PC time series of the oceanic and atmospheric datasets to uncover their underlying patterns of statistical dependence. Whereas simple linear regression relates two variables and multiple regression relates one set of independent variables 
to one dependent variable, CCA finds relationships between two sets of variables where neither set is defined as the dependent variable set (Hotelling 1936). A linear combination of original variables from one dataset is formed that is related the strongest, via correlation, with a linear combination of original variables formed from the other dataset. Once the first pair of linear combinations is formed that identifies the strongest association between each dataset, the variance explained by that pair is removed from each respective dataset and the procedure is repeated.

The association between the variables, that is, the strength of the canonical correlations, is a function of how many PCs are used as input for the CCA. The more PCs used for input, the stronger the canonical correlations become. However, this increase in the strength of the canonical correlation arises at the expense of adding more "noise" to the analysis, and the resulting spatial patterns account for less variance. A variety of guidance rules were thus used to objectively determine the number of PCs to retain for the CCA analysis - the rule $N$ test (Preisendorfer 1988), the Guttman criterion (Guttman 1954), and a scree test (Cattell 1966). CCA has proven useful in identifying coupled patterns of oceanic and atmospheric variability (e.g., Preisendorfer 1988; Livezey and Smith 1999; von Storch and Zwiers 1999). Each pattern of SST variability that emerges is thus, by construction, closely associated with an accompanying pattern of atmospheric circulation. The interdecadal Pacific signal (IPS) is the leading pattern of ocean-atmosphere interaction that emerges from the CCA, and it potentially exhibits unique interdecadal variability (Frauenfeld and Davis 2002); it is this signal, the IPS, that will be further evaluated here.

To determine the extent to which there are preferred time scales in the time series of a particular pattern such as the PDO or the IPS, we analyze the spectra of the annually averaged time series using the multitaper (MTM) spectral analysis method (Thomson 1982). MTM employs multiple data tapers to provide an optimally low-variance estimate of the spectrum of a time series. We make use of the Mann and Lees (1996; see also Ghil et al. 2002) implementation of the method, which provides a robust estimate of an assumed AR(1) "red" noise background of the spectrum of the time series. As red noise arguably represents the simplest null hypothesis that can be motivated for stochastic atmospheric and/or oceanographic processes, this estimate provides the basis for testing whether or not features can be identified in the spectrum that are statistically significant relative to the null hypothesis of stochastic variability. If no such features are evident, it is reasonable to consider the underlying time series as being consistent with the realization of a simple stochastic process. On the other hand, features that are significant relative to this null hypothesis may be more interesting from a climatic point of view (see, e.g., Mann and Lees 1996).

If the leading pattern of ocean-atmosphere variability indeed represents a unique interdecadal component of climate variability of the Pacific Ocean, then removal of that pattern from the leading mode (PC) of SST variability should leave only interannual and shorterscale SST variability. Using least squares linear regression, the relationship between the interdecadal signal and the leading SST mode is established and subsequently removed from this leading SST PC. The residual variability is then further evaluated to examine how it relates to Pacific climate.

One issue that arises when presenting an interdecadal pattern in an analysis using only a 52-yr data record is the extent to which $52 \mathrm{yr}$ of data can really capture interdecadal variability. To explore this issue and obtain a sense of how the interdecadal variability is manifest on a century scale, the time series of the IPS is projected onto the entire available 1856-2002 data record of the seasonally standardized Kaplan SST anomalies (because upper-atmospheric data are not available before the late 1940s, the CCA cannot be performed for 1856-2002). The resulting time series is then plotted and multitaper spectral analysis is applied to determine if the 1856-2002 time series still exhibits high power at interdecadal scales.

A number of studies have hypothesized that changes in the extratropical Pacific drive lower-frequency climate variability (e.g., Latif and Barnett 1994). Therefore, the entire PCA-CCA and multitaper spectral analysis methodology is repeated for the tropical and extratropical Pacific separately to explore the origins of the interdecadal climate signal.

\section{Interdecadal Pacific signal}

The leading pattern produced by the CCA between Pacific SSTs and the Northern Hemisphere circumpolar vortex is, by its statistical construction, a joint pattern of interaction. The strength of the relationship between the atmospheric and oceanic components is described by a canonical correlation of 0.71 ; the SST pattern accounts for $7.5 \%$ of the variance of the Pacific SST field, and the atmospheric pattern accounts for $6.3 \%$ of the variance in the Northern Hemisphere vortex (Frauenfeld and Davis 2002). As pointed out in Frauenfeld and Davis (2002), however, these numbers are merely a function of how many PCs are retained as input for the 
CCA. A higher canonical correlation is achieved at the expense of variance explained, and vice versa. Additionally, we do not argue that the IPS is a major new pattern accounting for substantial amounts of variance, but instead that it is a signal embedded in the climate system of the Pacific Ocean.

The time series of this joint ocean-atmosphere signal, the IPS, is predominantly negative from the beginning of the record until the Pacific Climate Shift of 1976/77 (Fig. 1, top). Since the climate shift, the time series has been almost exclusively positive. Prior to the 1976/77 shift, the IPS was temporally similar to the PDO (Fig. 1, bottom), exhibiting similar positive and negative anomalies, though different in magnitude. However, the IPS's distinct positive state since 1976/77 clearly distinguishes it from the PDO, especially the period from 1990 to 2000 and the unprecedented positive anomalies during the late 1990s. Owing to a significant component of interannual variability, the PDO exhibits multiple negative excursions throughout these last two decades and perhaps has exhibited a major phase shift in the mid-1990s, whereas the IPS has not. In fact, a number of studies have suggested that another
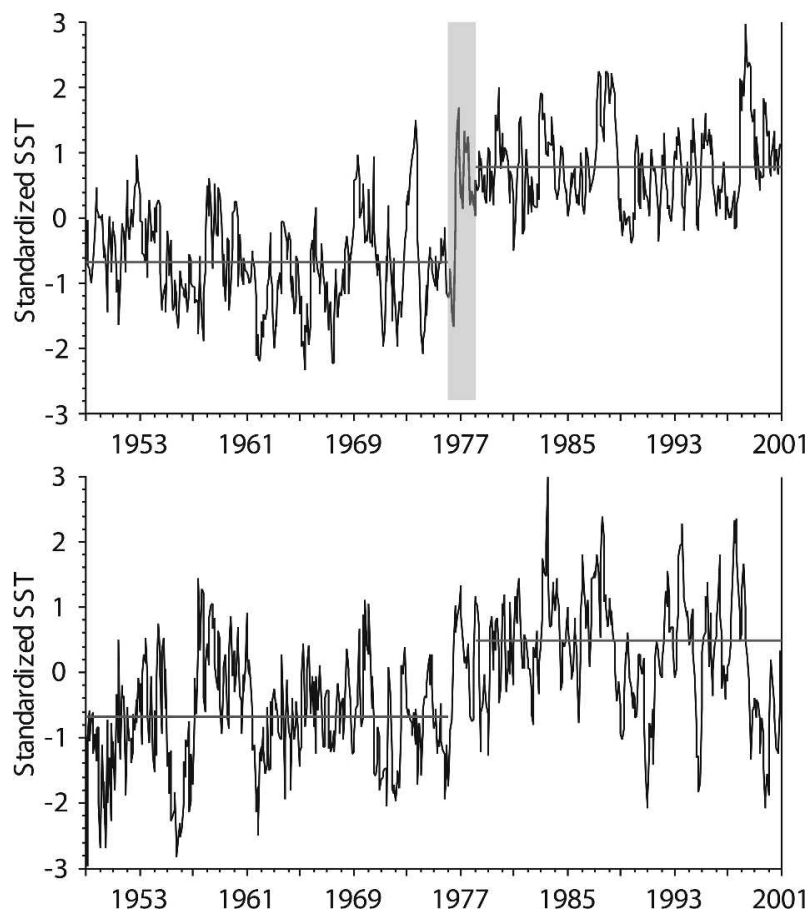

FIG. 1. Time series of the (top) IPS and (bottom) PDO. The approximate time of the Pacific Climate Shift is designated by the shaded area on each figure. Whereas the IPS time series has remained positive since $1977(\bar{x}=+0.78)$, the PDO $(\bar{x}=+0.49)$ exhibits numerous negative anomalies and perhaps a regime shift toward negative values in the late 1990s. The pre-1976 mean anomalies for the IPS and PDO are both -0.68 .
PDO regime shift occurred around 1989, although this later event differed in its climatic and ecosystem characteristics compared to 1976/77 (e.g., Hare and Mantua 2000). It has also been argued that the post-1976/77 regime has persisted through at least 1997 (Mantua et al. 1997; Mantua and Hare 2002). Analysis of the IPS time series, which has much less interannual-scale variability than the PDO index, suggests that there has not been a regime shift in interdecadal Pacific SST variability through at least 2000 .

The positive phase of the IPS, which has dominated since 1976/77, is spatially similar to the PDO. Most of the extratropical North Pacific is described by belownormal SSTs, whereas the Tropics are characterized by positive anomalies that extend northward along the west coast of North America (Fig. 2). It is important to reiterate that while the $\mathrm{PDO}$ is calculated for extratropical SSTs only (north of $20^{\circ} \mathrm{N}$ ), the IPS is calculated for $25^{\circ} \mathrm{S}-65^{\circ} \mathrm{N}$. The PDO and IPS differ in that the IPS also has positive anomalies in the western subtropical Pacific off the east coast of Asia in the vicinity of the so-called "Kuroshio Extension," and the North Pacific negative anomaly wraps around the positive Kuroshio
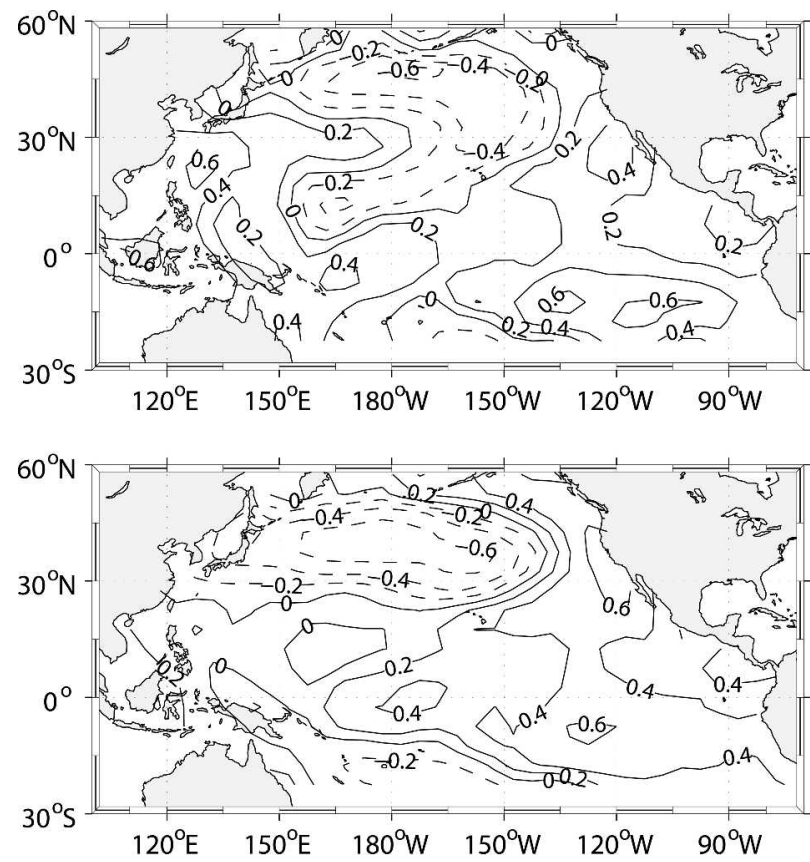

FIG. 2. SST anomalies associated with the (top) IPS and (bottom) PDO. The PDO is constructed using SSTs north of $20^{\circ} \mathrm{N}$ only, while the domain of the IPS extends from $25^{\circ} \mathrm{S}$ to $65^{\circ} \mathrm{N}$. Contours represent correlations between the patterns' time series and the original SST data. Positive correlations are solid lines; negative correlations are dashed lines; the contour interval is 0.2 . For the 624-month time series, correlations greater than 0.08 are statistically significant, and therefore all but the 0 contour are significant at the 0.05 alpha level. 


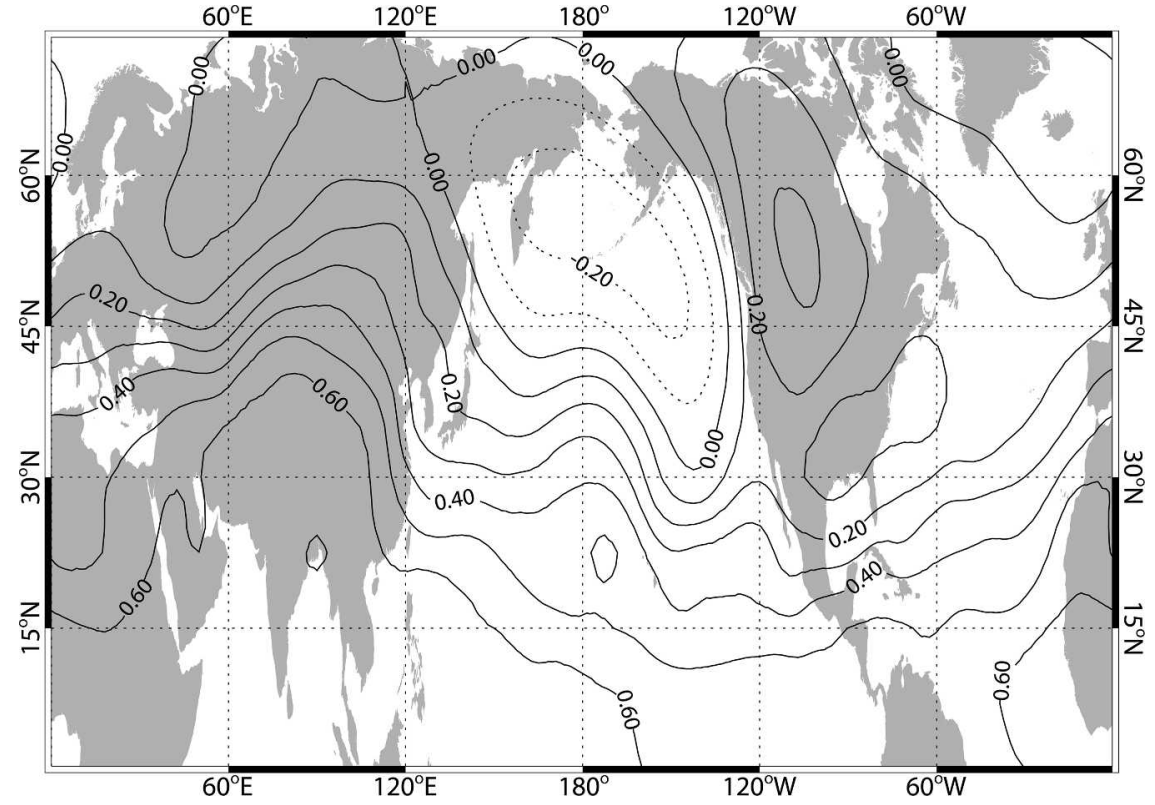

FIG. 3. The 500-hPa geopotential height pattern associated with the IPS's atmospheric component. Contours represent the correlations between the IPS time series and the seasonally standardized gridded 500-hPa geopotential height anomalies. Positive correlations are solid lines; negative correlations are dashed lines; the contour interval is 0.1. For the 624month time series, correlations greater than 0.08 are statistically significant, and therefore all but the 0 contour are significant at the 0.05 alpha level.

Extension region well into the tropical west Pacific (Fig. 2, top). The emergence of this feature in a decadal pattern lends support to the importance of changes in either the strength or location (Seager et al. 2001) of the gyre circulation in decadal climate variability. The anomaly feature in the IPS near the Kuroshio region is partially driven by the emergence of strong positive SST anomalies beginning around 1997, in conjunction with, or perhaps following, the major 1997-98 ENSO event. However, this Kuroshio feature is evident throughout the 1949-2000 period, more so before the 1976/77 shift than afterward.

The pattern of interdecadal atmospheric variability (Fig. 3) that arises jointly with the above SST pattern indicates that interdecadal Pacific SST variability is linked with atmospheric circulation upstream, over most of mid- to low-latitude Asia, as well as over southern Europe (Frauenfeld and Davis 2002). Positive geopotential height anomalies over mid- to low-latitude Eurasia are associated with positive SST anomalies in the Kuroshio region and the tropical Pacific, and with negative SST anomalies in the north-central Pacific, extending southwestward into the Tropics. Although the atmospheric pattern also illustrates the Aleutian low and Alberta high components of the Pacific-North American teleconnection pattern, these anomalies are weak ( $R=\sim 0.2)$ compared to the anomalies over Asia
$(R=0.4-0.7)$. Regions of strong variability in this interdecadal atmospheric pattern correspond to regions of strong trends observed in the Northern Hemisphere vortex, thereby indicating that the vortex has been contracted in the post-1976/77 regime over mid- to lowlatitude Eurasia in conjunction with a general atmospheric warming in that region (Frauenfeld and Davis 2003).

This mid- and low-latitude circulation anomaly region observed in both trends of the circumpolar vortex and this interdecadal ocean-atmosphere pattern may also be consistent with high-latitude atmospheric circulation trends described by the Arctic Oscillation in the Eurasian region, where it has been argued to account for $50 \%$ of the observed warming (Thompson et al. 2000). However, an argument could also be made for a pattern of zonally symmetric positive atmospheric anomalies across most of the Northern Hemisphere south of $20^{\circ} \mathrm{N}$ that reach a maximum over Asia. This could suggest that the atmospheric circulation changes could also be related to warming of the tropical atmosphere in response to observed tropical SST increases in recent decades, as captured by the IPS's positive western tropical Pacific anomalies. Either way, this statistical ocean-atmosphere linkage suggests that a key to both the 1976/77 regime shift and the decadal climate variability observed in the Pacific Ocean may lie in the 
upstream atmospheric circulation and the analogous general warming of the Tropics, or the general warming of the Eurasian landmass.

Multitaper spectral analysis indicates that the IPS time series exhibits a very clear secular peak indicative of low-frequency variability that is quite distinct from the null hypothesis of a red-noise stochastic process (Fig. 4, top). The spectral peak is highly statistically significant $(>99 \%)$, exhibiting spectral power more than 6 times greater than the estimated median rednoise background near zero frequency. Note, again, that with a 52-yr time series and time-frequency bandwidth of $w=2 \mathrm{~N}$ as employed in the spectral analysis, oscillatory behavior on time scales longer than $26 \mathrm{yr}$ is indistinguishable from the trend. This low-frequency peak therefore corresponds to interdecadal variability on time scales longer than $20 \mathrm{yr}$, that is, either an oscillation or a secular trend. There is, furthermore, a
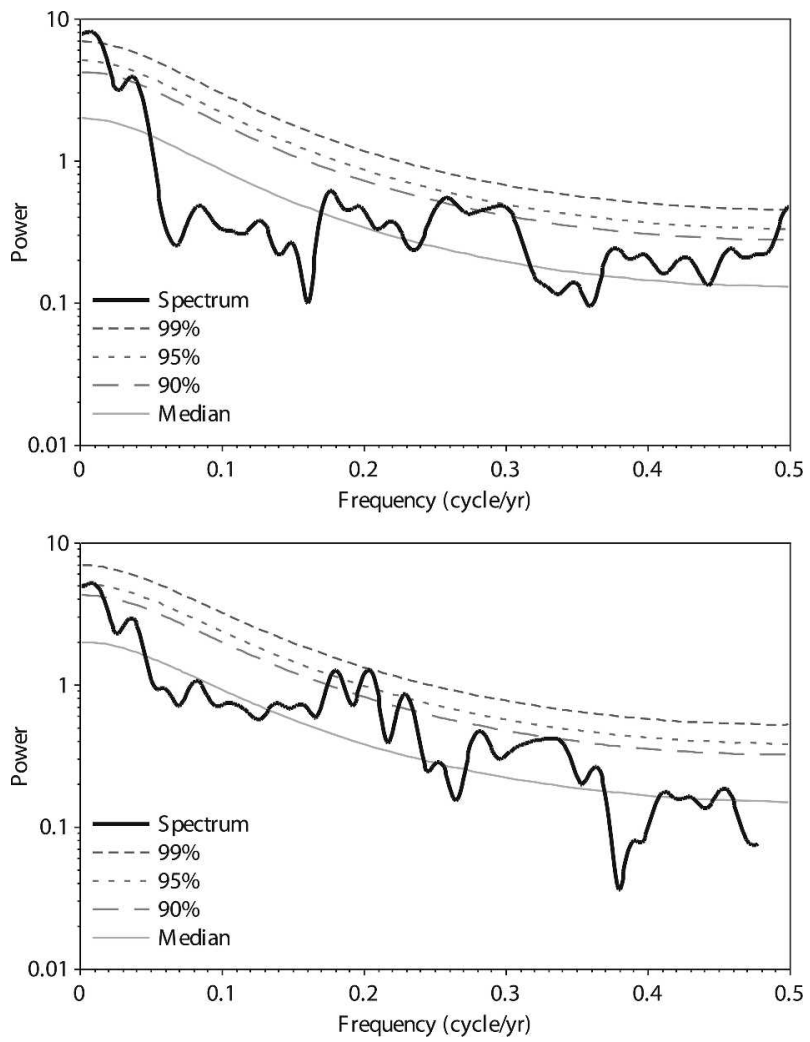

FIG. 4. Multitaper spectrum and red-noise confidence intervals based on the robust background noise estimation procedure of Mann and Lees (1996) for the annual (Jan-Dec average) time series of the (top) IPS and (bottom) PDO. While the IPS exhibits a highly significant secular peak (relative to a red-noise null hypothesis) indicative of enhanced interdecadal variability and no statistically significant variability on the $3-7-y$ r interannual time scales typical of ENSO, the PDO only exhibits enhanced variability on interannual time scales. clear break point in the spectrum between the higherfrequency variance, which falls within the red-noise background, and the low-frequency variance $(<0.07$ cycles $\mathrm{yr}^{-1}$ ), that is, the interdecadal variance. No significant variability is evident at shorter time scales; therefore the IPS is a truly interdecadal signal that, unlike the PDO, does not contain a component of significant interannual variability.

By comparison, the PDO time series exhibits only a slightly significant $(95 \%$ level) secular peak, but it mainly displays significant peaks around the 0.2 cycles $\mathrm{yr}^{-1}$ frequency that are indicative of interannual variability (Fig. 4, bottom). There are no features in the spectrum that exceed the $99 \%$ significance level relative to the null hypothesis of red noise. The PDO series thus appears to represent, in large part, the behavior of a stochastic red-noise process with perhaps modest contributions from interdecadal and interannual components of climate variability.

The leading pattern/PC of the Pacific SST field includes both interannual and interdecadal variability, similar to the PDO. If we have indeed isolated an interdecadal signal in Pacific SSTs, then removal of this signal, that is, the IPS, from the leading SST PC should leave a time series dominated by interannual ENSOtype variability. Similar to the analyses of Zhang et al. (1997) and Enfield and Mestas-Nuñez (1999), we removed the interdecadal variability from the leading SST PC via linear regression to test this hypothesis. The time series of the IPS is regressed against the time series of the leading SST PC, and the residuals are compared to the Niño-3.4 SST index. The residual variability of the leading SST PC after the IPS time series is removed produces a time series that is, essentially, the time series of El Niño (Fig. 5) and correlates highly with the Niño-3.4 SST time series $(R=0.86 ; p<0.001)$. This

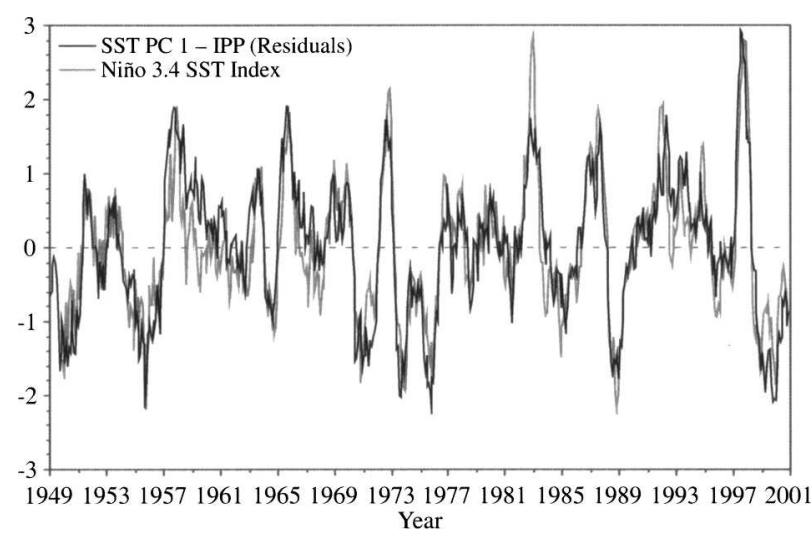

FIG. 5. Time series of the SST PC1 minus IPS residuals and the Niño-3.4 index. The two time series are correlated at $73 \%(R=$ $0.85 ; p<0.001$ ). 
underscores the remarkably clean nature of the separation of interannual ENSO-related variability and distinctly interdecadal ocean-atmosphere variability that is afforded by use of the IPS.

To gain further insight into this interdecadal pattern's long-term variability, the 1856-2002 IPS time series was created (Fig. 6). This time series indicates an oscillatory behavior, such that the IPS was increasing until the late $1800 \mathrm{~s}$, decreasing until $\sim 1920$, increasing until the early 1940s, decreasing until the late 1960smid-1970s, and increasing thereafter, beginning with the 1976/77 regime shift. This time series also illustrates the uniqueness of the positive period beginning after the climate shift, as well as the unprecedented positive anomalies in the late 1990s. This unusual 1998-2002 period in the IPS, combined with the unprecedented 1998-2000 period in the time series of the second pattern produced by this CCA (not shown; see Fig. 7 of Frauenfeld and Davis 2002), may be related to the "perfect ocean for drought" reported by Hoerling and $\mathrm{Ku}$ mar (2003). Multitaper spectral analysis indicates that, like its 1949-2000 counterpart, the IPS for 1856-2002, as well as for 1856-1949, exhibits highly significant $(>99 \%)$ variability at interdecadal time scales (not shown).

\section{Tropical versus extratropical origins}

To explore the origins of the interdecadal oceanatmosphere interaction, the Pacific Ocean domain was split according to its patterns of tropical $\left(25^{\circ} \mathrm{S}-20^{\circ} \mathrm{N}\right)$ versus extratropical $\left(25^{\circ}-65^{\circ} \mathrm{N}\right)$ variability, and the PCA-CCA methodology was repeated on the vortex and the two SST domains separately. Both the tropical and extratropical spatial SST patterns of oceanatmosphere variability uncovered are very similar to

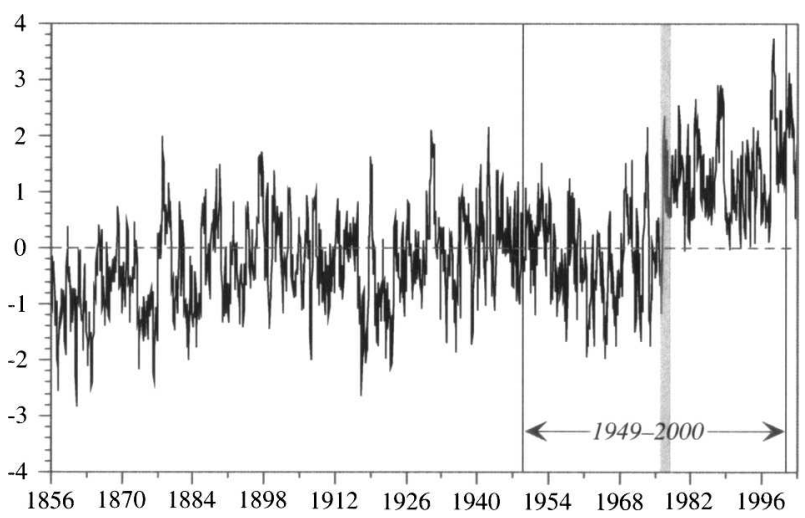

FIG. 6. Projection of the IPS onto 1856-2002 SST data. Indicated on this time series is also the original $1949-2000$ period (Fig. 1 , top) and the 1976/77 shift. that of the IPS. However, the time series suggest that the IPS has its origins in the Tropics, as the tropical time series is indicative of much greater year-to-year and decade-to-decade persistence than that of the extratropics (Fig. 7).

Performing a multitaper spectral analysis again using a robust background noise and significance estimation procedure on the two time series further indicates that the tropical time series indeed exhibits more prominent low-frequency variability than the extratropical time series (Fig. 8). Both the tropical and extratropical signals of ocean-atmosphere interaction exhibit highly significant secular peaks $(>99 \%)$, suggesting interdecadal variability on time scales greater than $20 \mathrm{yr}$. Again, the spectra demonstrate clear separation between higherand lower-frequency variability. At the low frequencies, the extratropical time series exhibits a spectral peak more than 8 times greater than the estimated median red-noise background. However, the tropical time series exhibits spectral power approximately 14 times greater at low frequencies, further suggesting that the roots of the interdecadal ocean-atmosphere variability perhaps lie in the Tropics.

Because the extratropical $25^{\circ}-65^{\circ} \mathrm{N}$ domain em-
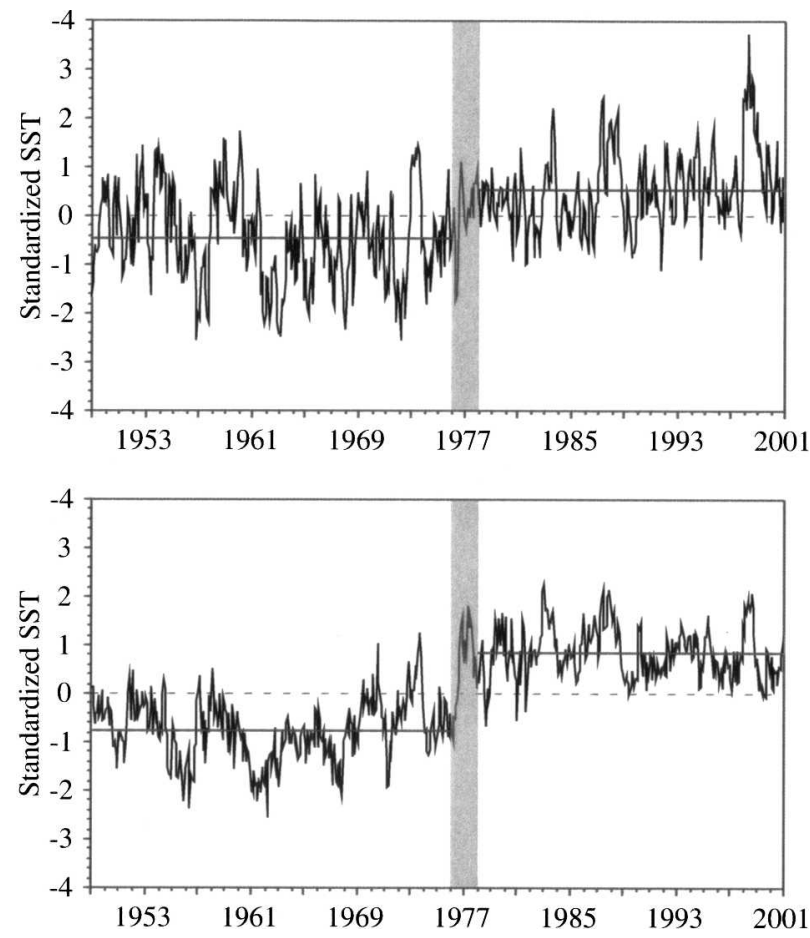

FIG. 7. As in Fig. 1, but for the (top) extratropical and (bottom) tropical ocean-atmosphere time series. The tropical IPS time series is much more exclusively negative prior to the 1976/77 shift $(\bar{x}$ $=-0.76)$ than the extratropical time series $(\bar{x}=-0.46)$, and more exclusively positive since the shift $(\bar{x}=+0.84)$ than the extratropical IPS $(\bar{x}=+0.54)$. 

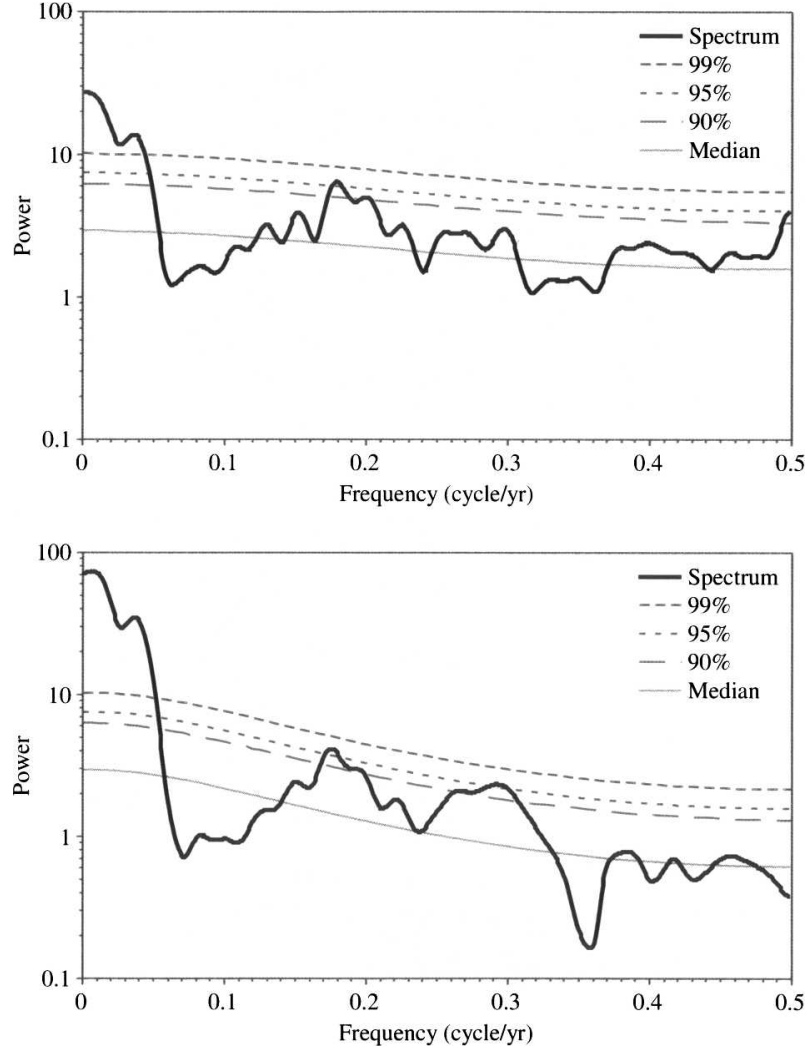

FIG. 8. As in Fig. 4, but for the (top) extratropical and (bottom) tropical ocean-atmosphere time series. While both exhibit a highly significant secular peak $(>99 \%)$ indicative of interdecadal variability, the tropical time series exhibits much greater spectral power at low frequencies.

ployed here corresponds closely to the domain of the PDO, comparing its spectrum (Fig. 8, top) with that of the PDO (Fig. 4, bottom) further demonstrates that the interdecadal ocean-atmosphere variability is substantially stronger than the PDO's. Although a slight interannual spectral peak also emerges, variability at this scale falls within the red-noise background, unlike the PDO's more prominent interannual spectral peak. The biennial spectral peak in the extratropical oceanatmosphere pattern corresponds to the Nyquist frequency and for this reason, the behavior of the spectrum near $f=0.5$ cycle $\mathrm{yr}^{-1}$ (2-yr period) should be disregarded because of the likelihood that it suffers from aliasing bias.

When performing linear statistical analyses such as $\mathrm{EOF} / \mathrm{PCA}$, the leading pattern is sometimes indicative of only any long-term trend that may be inherent in the data (e.g., Parker and Folland 1991). To determine if the IPS merely represents a long-term trend, the global SST anomalies were removed from the 1856-2002 IPS for each month similar to the approach of Zhang et al.
(1997). The resulting time series still exhibits highly significant $(>99 \%)$ secular/interdecadal variability, and the spatial pattern was largely unaffected (not shown). To further ensure that a trend component is not dominating the IPS, the long-term trend at each grid point was also separately subtracted from the spatial IPS, and the IPS was again recovered. This is true for the 18562002 period, as well as the subperiods of 1856-1948 and 1949-2002. We are therefore confident that the IPS does not merely represent a long-term trend. Similarly, although multitaper spectral analysis of a 52-yr time series cannot distinguish a secular trend from oscillatory behavior on time scales longer than $26 \mathrm{yr}$, this suggests that we are dealing with an interdecadal oscillation (see Fig. 6) more so than a secular trend.

\section{Conclusions}

We have identified a uniquely interdecadal signal embedded in the Pacific Ocean-Northern Hemisphere atmosphere system that, by its statistical construction, is representative of the interaction between the largescale atmospheric circulation of the Northern Hemisphere and SSTs of the Pacific Ocean. There is thus a pattern of unique low-frequency variability in Pacific SSTs when they are coupled with atmospheric circulation. Our interdecadal Pacific signal of oceanatmosphere interaction exhibits little of the PDO's higher-frequency variability and is characterized by a particularly prominent multiyear and multidecadal persistence. The time series of the IPS from 1949-2000dominated by the Pacific Climate Shift with negative anomalies prior to 1976/77 and almost exclusively positive anomalies since-is representative of interdecadal variability at time scales longer than $20 \mathrm{yr}$. Given the limited 52-yr length of our observational data record, this interdecadal variability could be indicative of an oscillation or a secular trend. Regardless, the IPS is characterized by much more prominent low-frequency variability and remarkable multiyear and multidecadal persistence than has been demonstrated for other $\mathrm{Pa}$ cific climate signals.

The conventional approach to large-scale climate index development, like that used to define the PDO pattern, employs methods like PCA on time series of spatial fields to identify large-scale variance changes. By contrast, our incorporation of a coupled oceanatmosphere decomposes the dominant pattern of SST variability into its interdecadal and interannual components, although it is possible that different physical processes are being captured here as compared to investigations of single spatial fields only.

Zhang et al. (1997) suggest that it is not possible to 
separate decade-to-century-scale variability from interannual variability using straightforward PCA and related techniques without high-pass and low-pass prefiltering of the time series, although multivariate frequency-domain methods have proven useful in this regard (e.g., Mann and Park 1996). We have essentially isolated the interdecadal variability of the Pacific Ocean from the interannual variability without the application of any frequency-domain filtering techniques, but instead by utilizing the coupled behavior of the Pacific Ocean with the Northern Hemisphere's atmospheric circulation. This implies that the interdecadal variability in the Pacific Ocean and the Northern Hemisphere atmosphere that is distinct from stochastic (red noise) variability arises from interactions between the ocean and atmosphere. The IPS represents a statistical linkage between the Pacific Ocean and the atmospheric circulation of the Northern Hemisphere. Such a statistical relationship may not be meaningful if no physical mechanism is present to account for such a relationship. It is the goal of our ongoing research to establish such a mechanism.

The time series of the IPS is the only empirically derived, distinctly interdecadal signal of Pacific Ocean SST variability. Although it is the PDO that is generally considered to be representative of decadal Pacific Ocean variability, the IPS does not contain any significant interannual variability and thus exhibits much greater multiyear and multidecadal persistence than the PDO. Furthermore, the PDO's spectral peaks are generally consistent with a red-noise background, whereas the IPS exhibits highly significant interdecadal variability. The IPS time series, therefore, seems to be a more faithful representation of interdecadal patterns of climate change in the Pacific Ocean-Northern Hemisphere atmosphere system. Because the residuals of the dominant pattern (PC) of Pacific SST variability after the IPS is removed exhibit essentially El Niño variability, the IPS itself contains little to no ENSO signal. However, the IPS nonetheless is linked more closely to tropical Pacific SST variability (i.e., tropical non-ENSO variability) than extratropical influences.

Our intent in this research is not to specifically challenge use of the PDO in climate change research-in fact, the IPS is likely a component of the PDO, and vice versa. Interdecadal Pacific variability has certainly been described in the literature by many researchers. For instance, Zhang et al. (1997) employed high- and lowpass filtering to partition the Pacific's leading mode of SST variability into interannual and interdecadal components. Removing their high-pass-filtered cold tongue index (which represents ENSO variability) from the global SST field results in a time series indicative of interdecadal variability and a spatial pattern, both similar to the PDO and the IPS, but without any variability in the Kuroshio region. Similarly, Enfield and MestasNuñez (1999) uncover interdecadal Pacific variability after removing a high- and low-pass-filtered component (ENSO) from low-pass-filtered SST data, although this SST variability still contains significant interannual variability. The spatial pattern is again similar to the PDO and the IPS, with the notable exception of the Kuroshio anomaly.

What separates these analyses and others from ours is not only our coupling of the ocean with the atmosphere, but our ability to identify interannual versus interdecadal variability without the use of data filtering. Apparently, the partitioning into interannual and interdecadal variability arises naturally from that coupling of the ocean with the atmosphere. Furthermore, the IPS, as demonstrated by the spectral analysis, contains virtually no significant interannual variability and is therefore only indicative of highly significant interdecadal variability on time scales of $20+$ years. Our approach of examining coupled ocean-atmosphere patterns represents a much cleaner separation of interannual and interdecadal variability. Furthermore, it represents an advance in Pacific SST climate studies by illustrating a direct statistical link between interdecadal Pacific variability and the atmospheric circulation over the low- to midlatitude Eurasian landmass.

Whereas our skill in understanding and predicting interannual ENSO-type fluctuations in the Pacific has made great advances in recent decades, our understanding of decadal-scale variability remains limited. This identification and isolation of the truly decadal component of the ocean-atmosphere system of the Pacific from the higher-frequency behavior should lead to better understanding and predictability of interdecadal climate variability and have widespread ramifications for the large marine ecosystems of the North Pacific Ocean and other climate-sensitive natural resources in the Pacific and North America.

Acknowledgments. We thank Dr. Adam Burnett for providing the circumpolar vortex data. We also thank the anonymous reviewers, whose comments improved this manuscript, and Dr. Martin Hoerling for his guidance as editor of this manuscript. This work was partly supported by the U.S. National Science Foundation through NSF Grant OPP-0229766.

\section{REFERENCES}

Alexander, M. A., I. Bladé, M. Newman, J. R. Lanzante, N.-C. Lau, and J. D. Scott, 2002: The atmospheric bridge: The in- 
fluence of ENSO teleconnections on air-sea interaction over the global oceans. J. Climate, 15, 2205-2231.

Angell, J. K., 1998: Contraction of the 300 mbar north circumpolar vortex during 1963-1997 and its movement into the eastern hemisphere. J. Geophys. Res., 103, 25 887-25 893.

- 1999: Comparison of surface and tropospheric temperature trends estimated from a 63-station radiosonde network 19581998. Geophys. Res. Lett., 26, 2761-2764.

_ - and J. Korshover, 1977: Variation in size and location of the $300 \mathrm{mb}$ north circumpolar vortex between 1963 and 1975. Mon. Wea. Rev., 105, 19-25.

Barnett, T. P., L. Dümenil, U. Schlese, E. Roeckner, and M. Latif, 1989: The effect of Eurasian snow cover on regional and global climate variations. J. Atmos. Sci., 46, 661-685.

Burnett, A. W., 1993: Size variations and long-wave circulation within the January Northern Hemisphere circumpolar vortex: 1946-89. J. Climate, 6, 1914-1920.

Cattell, R. B., 1966: The scree test for the number of factors. $J$. Multiv. Behav. Res., 1, 245-276.

Cohen, J., and D. Entekhabi, 1999: Eurasian snow cover variability and Northern Hemisphere climate predictability. Geophys. Res. Lett., 26, 345-348.

Enfield, D. B., and A. M. Mestas-Nuñez, 1999: Multiscale variabilities in global sea surface temperatures and their relationships with tropospheric climate patterns. J. Climate, 12, 27192733.

Frauenfeld, O. W., and R. E. Davis, 2000: The influence of El Niño-Southern Oscillation events on the northern hemisphere $500 \mathrm{hPa}$ circumpolar vortex. Geophys. Res. Lett., 27, $537-540$.

_ and — 2002: Midlatitude circulation patterns associated with decadal and interannual Pacific Ocean variability. Geophys. Res. Lett., 29, 2221, doi:10.1029/2002GL015743.

— trends and climate change implications. J. Geophys. Res., 108, 4423, doi:10.1029/2002JD002958.

Ghil, M., and Coauthors, 2002: Advanced spectral methods for climatic time series. Rev. Geophys., 40, 1003, doi:10.1029/ 2000RG000092.

Guilderson, T. P., and D. P. Schrag, 1998: Abrupt shift in subsurface temperatures in the tropical Pacific. Science, 281, 240243.

Guttman, L., 1954: Some necessary conditions for common-factor analysis. Psychometrika, 19, 149-161.

Hare, S. R., and N. J. Mantua, 2000: Empirical evidence for North Pacific regime shifts in 1977 and 1989. Progress in Oceanography, Vol. 47, Pergamon Press, 103-145.

Hasselmann, K., 1976: Stochastic climate models. Part I: Theory. Tellus, 28, 473-485.

Hoerling, M. P., and A. Kumar, 2003: The perfect ocean for drought. Science, 299, 691-694.

Hotelling, H., 1936: Relations between two sets of variables. Biometrika, 28, 321-377.

Kaplan, A., M. Cane, Y. Kushnir, A. Clement, M. Blumenthal, and B. Rajagopalan, 1998: Analyses of global sea surface temperature 1856-1991. J. Geophys. Res., 103, 18 567-18 589.

LaSeur, N. E., 1954: On the asymmetry of the middle-latitude circumpolar current. J. Meteor., 11, 43-75.
Latif, M., and T. P. Barnett, 1994: Causes of decadal climate variability over the North Pacific and North America. Science, 266, 634-637.

Levitus, S., J. I. Antonov, T. P. Boyer, and C. Stephens, 2000: Warming of the World Ocean. Science, 287, 2225-2229.

Livezey, R. E., and T. M. Smith, 1999: Covariability of aspects of North American climate with global sea surface temperatures on interannual to interdecadal timescales. J. Climate, 12, 289302.

Mann, M. E., and J. M. Lees, 1996: Robust estimation of background noise and signal detection in climatic time series. Climatic Change, 33, 409-445.

— temperature and sea level pressure variability in the Northern Hemisphere during the last century. J. Climate, 9, $2137-$ 2162

Mantua, N. J., and S. R. Hare, 2002: The Pacific Decadal Oscillation. J. Oceanogr., 58, 35-44.

— — _ Y Y. Zhang, J. M. Wallace, and R. C. Francis, 1997: A Pacific interdecadal climate oscillation with impacts on salmon production. Bull. Amer. Meteor. Soc., 78, 1069-1079.

Markham, C. G., 1985: A quick and direct method of estimating mean monthly global temperatures from $500 \mathrm{mb}$ data. Prof. Geogr., 37, 72-74.

Miller, A. J., D. R. Cayan, T. P. Barnett, N. E. Graham, and J. M Oberhuber, 1994: The 1976-77 climate shift of the Pacific Ocean. Oceanography, 7, 21-26.

Minobe, S., 1997: A 50-70 year climatic oscillation over the North Pacific and North America. Geophys. Res. Lett., 24, 683-686.

_ 1999: Resonance in bidecadal and pentadecadal climate oscillations over the North Pacific: Role in climatic regime shifts. Geophys. Res. Lett., 26, 855-858.

Namias, J., 1976: Some statistical and synoptic characteristics associated with El Niño. J. Phys. Oceanogr., 6, 130-138.

Parker, D. E., and C. K. Folland, 1991: Worldwide surface air temperature trends since the mid-19th century. GreenhouseGas Induced Climatic Change: A Critical Appraisal of Simulations and Observations, M. E. Schlesinger, Ed., Elsevier, 173-193.

Preisendorfer, R. W., 1988: Principal Component Analysis in Meteorology and Oceanography. Elsevier, $425 \mathrm{pp}$.

Seager, R., Y. Kushnir, N. H. Naik, M. A. Cane, and J. Miller, 2001: Wind-driven shifts in the latitude of the KuroshioOyashio Extension and generation of SST anomalies on decadal timescales. J. Climate, 14, 4249-4265.

Thompson, D. W. J., J. M. Wallace, and G. C. Hegerl, 2000: Annular modes in the extratropical circulation. Part II: Trends. J. Climate, 13, 1018-1036.

Thomson, D. J., 1982: Spectrum estimation and harmonic analysis. Proc. IEEE, 70, 1055-1096.

von Storch, H., and F. W. Zwiers, 1999: Statistical Analysis in Climate Research. Cambridge University Press, 484 pp.

Willett, H. C., 1949: Long-period fluctuations of the general circulation of the atmosphere. J. Meteor., 6, 34-50.

Zhang, Y., J. M. Wallace, and D. S. Battisti, 1997: ENSO-like interdecadal variability: 1900-93. J. Climate, 10, 1004-1020. 